\title{
Diffusion based homogenization method for 1D wave propagation
}

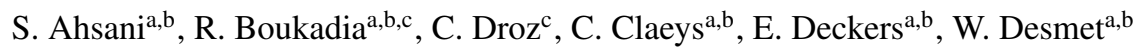 \\ ${ }^{a}$ KU Leuven, Department of Mechanical Engineering, Division PMA, Celestijnenlaan 300 - box 2420, Heverlee, Belgium \\ ${ }^{b}$ DMMS Lab, Flanders Make, Heverlee, Belgium \\ ${ }^{c}$ Ecole Centrale de Lyon, 36 Avenue Guy de Collongue, 69134 Ecully Cedex, France
}

\begin{abstract}
The implementation of increasingly complex periodic structures for vibro-acoustic purposes in civil engineering and transportation industry creates new modeling and computational challenges, mainly due to the multi-scale nature of the structures. Homogenization techniques able to describe the local dynamics effects appearing in periodic structures have therefore received significant attention in the past years. In this paper, a homogenization technique is proposed for $1 \mathrm{D}$ periodic media, where the equivalent material properties are determined using the local wave scattering characteristics of the periodic medium. A Wave Finite Element scheme is used to retrieve the multi-modal interface diffusion coefficients based on a unit-cell FE model of the periodic structure. The homogenized model is then used to derive wavenumbers and Frequency Response Function of the waveguide. Four examples are proposed, including a 3D periodic waveguide exhibiting locally resonant bandgaps. Good agreement is observed. Also limitations of the method are discussed.

Keywords: Periodic Structures, Homogenization, Wave Finite Element, Diffusion Matrix Model, Coherent Potential Approximation
\end{abstract}

Email address: sepide.ahsani@kuleuven. be (S. Ahsani) 


\section{Introduction}

Periodic structures are encountered in many industries, including civil, automotive and aerospace. These periodic configurations (e.g. honeycomb, stiffened, perforated) can be found in most lightweight panels due to their well-known stiffness-to-weight ratio. Although the research on this topic has been largely driven by phononic and photonic crystals [1, 2, 3] and metamaterials [4, 5, 6], an extensive work has also been dedicated to enhance the micro-architectures of lightweight structures for low-frequency or vibroacoustic purposes [7]. The development of structurally advanced materials, often exhibiting strong multi-scale behavior, has been responsible for a variety of modelling challenges, especially in the mid-frequency range, or equivalently sub-wave length regime. Periodic structure theory, and the Wave Finite Element framework [8] in particular, have been exceptionally efficient at handling large FE-modelled 3D periodic structures [9].

Although the periodic structure theory has been applied successfully to polar, curved or coupled periodic structures [10], its implementation is rarely exempt from numerical issues [11] or high computational costs when industrialscaled assemblies are considered. Therefore, reducing the size of the problem using two general methods, namely, model order reduction and dynamic homogenization, has received a regain of attention over the past years.

In the first category, recent developments by Droz et al. [12] can be cited. In which, a reduced formulation for the Wave Finite Element Method (WFEM), involving a projection of the periodic state vectors on a reduced subset of Bloch waves, have been proposed. Moreover, Boukadia et al. [13] used an inverse approach to compute the Bloch solutions, leading to even higher performances, even in the presence of evanescent waves. Additionally, Mencik et al. [14] also developed a reduction framework for computing the forced response of waveguides by truncating the Bloch expansion to the most significant modes. These developments, among many others, reveal the current numerical challenges.

In the second category, asymptotic approaches are often used [15], which include the micro-scale behavior in the elasto-dynamic equation of the system such that the solution contains both macro- and micro-scale components. These approaches are valid under the main assumption that the lengthscale of the unit cell (micro-scale) is much smaller than the wavelength (macro-scale) in the medium. In recent developments, Fossat et al. [16] were able to apply the asymptotic homogenization on highly contrasted structures and yet capture their unique behavior such as inner resonance behavior. So far, the advantages of these methods are highlighted in many applications [17, 18]. A closed analytical expression is however required, which may limit the range of possible periodic configurations to the ones that are not geometrically too complex such that the dynamic behavior can be predicted analytically.

Ensemble averaging is another homogenization technique available in literature, which was developed by Willis [19], who considered an effective (average) response to relate the effective stress and momentum to the effective strain and velocity of the medium under certain harmonic wave. As a result, the dispersion curve can be captured, but the approach is only valid in low frequencies.

Another possible way to create an equivalent medium is to base the definition of the homogenized medium on the 
similarity between its local scattering characteristics and the ones of the original structure. As the Coherent Potential Approximation (CPA) method proposed by Wu et al. [20], in which they consider a cylindrical scatterer embedded in a solid host medium as their unit cell, assuming it is surrounded by an equivalent medium. They calculate the effective properties by minimizing the scattering coefficients at the interface of the unit cell and its equivalent medium. There are two limitation to this method. The first one is the dependency of the method on analytical description of scattering coefficients. The second one is that the size of the unit cell should be much smaller than the wavelength in both host and effective medium.

The above mentioned methods are either based on analytical descriptions of the unit cell's dynamic behavior, or are limited by the wavelength-to-unit-cell size ratio. Therefore, they cannot accurately predict the original model's behavior when the geometry is too complex to allow calculation by analytical means. For example, replacing a 3D resonator by a tuned vibration absorber (TVA) could be categorized as a homogenization approach but to determine the effective mass of the TVA is not always straightforward [21], since the overall mass of the resonator does not always contribute to the effective mass of the TVA. Another example could be the asymptotic technique applied on periodic ribbed plate in [16], where the Euler-Bernoulli beam theory and Love-Kirchhoff plate theory are expanded to higher order and the coupling condition between the two is derived considering the scale separation. Although this technique is very efficient and gives an explicit form for the dynamic behavior of such structure, it is valid under the assumed geometry. In this paper, a dynamic homogenization technique is developed that exploits WFEM framework in a CPA context to create an equivalent media in order to overcome these issues. In the proposed technique, the Diffusion Matrix Model (DMM) [22, 23, 24], which is an highly effective numerical technique to determine the local scattering properties of Bloch waves propagating through a FE-modelled interface, is used.

First, an analytical theory that allows to accurately represent the physical behaviour of the structure under study (torsion, bending, compression) is selected. This theory informs us about the nature of the parameters that should be updated to create the homogenized model. Afterwards, the homogenized model is identified by solving an optimization involving its scattering matrix, whereby the reflection coefficients are minimized and the transmission coefficients mimic the phase shift that would occur through a unit cell of the periodic medium. The diffusion based homogenization framework is general and while in this paper the focus is on Euler-Bernoulli beams, more complex analytical models [25] could be used (e.g. Timoshenko beam, third-order shear beam theories) and coupled to more complex geometrical unit cells as long as their kinematic equations are known, and incompatible meshes are coupled with approaches such as Lagrange Multipliers [22]. Coupling incompatible meshes requires intrusive manipulation of the FEM package used. Therefore, for implementational reasons, this paper focuses on Euler-Bernoulli beam theory, which leads to identical cross-sections between the homogenized and the original model in order to check the validity of the proposed approach. Consequently, the proposed method can capture one or multi-modal behavior depending on the analytical theory considered for the coupling element regardless of geometrical complexity. At the end, the homogenized model is used as a representation of the real structure to derive the wavenumbers and frequency response functions (FRFs) of the waveguide. 
The rest of the paper is structured as follows: In Section 2, the WFEM formulation is recalled and the method to compute the diffusion characteristics of a periodic waveguide is described. Afterwards, the optimization algorithm is detailed, where a reduced-order modeling strategy is combined with the Bloch modeling technique. In Section 3 , the validity of the method is investigated to retrieve the local wave dispersion characteristics from the homogenized waveguide. Four numerical examples are considered: a 2-degrees-of-freedom (DOF) beam model as a proof-ofconcept and three 3D FE-modeled waveguides with stopband behavior. Furthermore, the same equivalent media is used to compute the dynamic response of a semi-finite and finite waveguide subjected to punctual excitation. Finally, the paper concludes with a main summary of the performances and accuracy of the method.

\section{Methodology}

To be able to explain the diffusion based homogenization, firstly the Diffusion Matrix Model (DMM), which incorporates the WFEM [26], is reviewed. Therefore, the diffusion based homogenization is explained in three parts. The first part reviews the Wave Finite Element method for 1D wave propagation, while the second part explains DMM. In the last part, a detailed description of the diffusion based homogenization method is provided.

\subsection{Wave Finite Element Method for $1 D$ waveguides}

This method describes wave propagation in periodic structures that are constructed by repeating a unit cell (UC) along one direction, see Figure 1

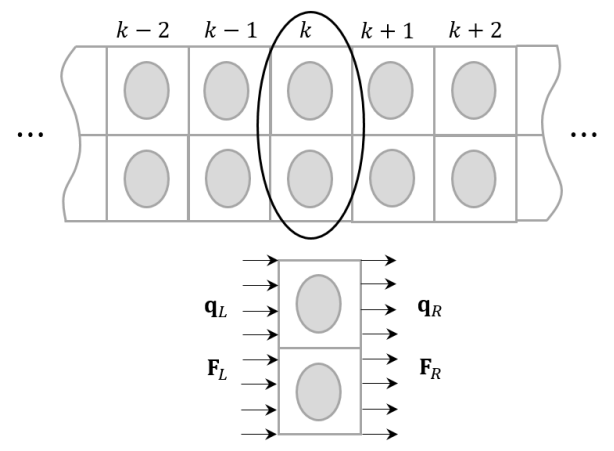

Figure 1: Schematic view a 1D periodic structure and its unit cell

The first step is to consider the finite element (FE) model of the $k^{\text {th }} \mathrm{UC}$ of the structure and discretizing its right $\left(\bullet_{R}\right)$ and left $\left(\bullet_{L}\right)$ boundary, see Figure 1 into $2 n$ degrees of freedom in terms of field variables:

$$
\mathbf{u}_{L}^{(k)}=\left[\begin{array}{c}
\mathbf{q}_{L}^{(k)} \\
-\mathbf{F}_{L}^{(k)}
\end{array}\right], \quad \mathbf{u}_{R}^{(k)}=\left[\begin{array}{c}
\mathbf{q}_{R}^{(k)} \\
\mathbf{F}_{R}^{(k)}
\end{array}\right] .
$$

In the definition above, $\mathbf{q}$ is the $n \times 1$ vectors of displacements, and $\mathbf{F}$ is vectors of forces of the same dimension. Considering the equation of motion, one can derive the transfer matrix $(\mathbf{S})$ of the $k^{\text {th }} \mathrm{UC}$, which relates the field variables at left and right boundary to each other: 


$$
\mathbf{u}_{R}=\mathbf{S} \mathbf{u}_{L},
$$

where the transfer matrix is defined as:

$$
\mathbf{S}=\left[\begin{array}{cc}
-\left(\mathbf{D}_{L R}\right)^{-1} \mathbf{D}_{L L} & -\left(\mathbf{D}_{L R}\right)^{-1} \\
\mathbf{D}_{R L}-\mathbf{D}_{R R}\left(\mathbf{D}_{L R}\right)^{-1} \mathbf{D}_{L L} & -\mathbf{D}_{R R}\left(\mathbf{D}_{L R}\right)^{-1}
\end{array}\right]
$$

In the equation above $\mathbf{D}$ is the condensed dynamic matrix of the $\mathrm{UC}$, and is defined as:

$$
\mathbf{D}\left[\begin{array}{l}
\mathbf{q}_{L} \\
\mathbf{q}_{R}
\end{array}\right]=\left[\begin{array}{l}
\mathbf{F}_{L} \\
\mathbf{F}_{R}
\end{array}\right] \quad \text { with } \quad \mathbf{D}=\mathbf{D}_{B B}^{*}-\mathbf{D}_{B I}^{*}\left(\mathbf{D}_{I I}^{*}\right)^{-1} \mathbf{D}_{I B}^{*},
$$

where subscripts $B$ and $I$ refer to the boundary (left and right) degrees of freedom, and the internal degrees of freedom, respectively. Moreover, the matrix $\mathbf{D}_{B B}^{*}$ is defined as:

$$
\mathbf{D}_{B B}^{*}=\left[\begin{array}{ll}
\mathbf{D}_{L L}^{*} & \mathbf{D}_{L R}^{*} \\
\mathbf{D}_{R L}^{*} & \mathbf{D}_{R R}^{*}
\end{array}\right] .
$$

The dynamic stiffness matrix is expressed as:

$$
\mathbf{D}^{*}=\mathbf{K}+j \omega \mathbf{C}-\omega^{2} \mathbf{M}
$$

where $\mathbf{K}$ is the stiffness matrix, $\mathbf{C}$ is the damping matrix, and $\mathbf{M}$ is the mass matrix.

Considering the continuity conditions at the interface of two consecutive UCs, and applying the Bloch-Floquet periodic boundary condition, we arrive to the following eigenvalue problem (EVP):

$$
\mathbf{S} \boldsymbol{\phi}_{i}=\mu_{i} \boldsymbol{\phi}_{i}
$$

where $\mu_{i}$ is the propagation parameter. Moreover, the vectors $\phi_{i}, i \in\{1, \ldots, 2 n\}$, are used as the basis of field variables: $\mathbf{u}_{L}^{(k)}=\boldsymbol{\Phi} \boldsymbol{Q}^{(k)}, \mathbf{u}_{R}^{(k)}=\boldsymbol{\Phi} \boldsymbol{Q}^{(k+1)}$.

This EVP (7) is ill-conditioned. Therefore, the alternative method proposed by Zhong and William [27] is used in this paper to improve the formulation:

$$
\left(\mathbf{N}-\mu_{i} \mathbf{L}\right)\left[\begin{array}{c}
\mathbf{q}_{L}^{(k)} \\
\mathbf{q}_{R}^{(k)}
\end{array}\right]=\mathbf{0} .
$$

The description of $\mathbf{N}$ and $\mathbf{L}$ can be found in [27]. The $2 n$ solutions of the EVP are divided in two groups. The first one corresponds to right going waves $\left(\bullet^{+}\right)$and the second group represents left going waves $\left(\bullet^{-}\right)$. Both groups can be separated from each other using the criteria suggested in [28]:

$$
\left|\mu_{i}^{+}\right|<1 \quad \text { and } \quad\left|\mu_{i}^{-}\right|>1 \text {. }
$$


For the propagation constants close to 1, energy flow can be used as separation criteria:

$$
\begin{aligned}
& \text { if }\left|\mu_{i}^{+}\right| \approx 1 \quad \text { then } \quad \operatorname{Re}\left(j \omega \boldsymbol{\phi}_{q} \cdot \boldsymbol{\phi}_{F}\right)>0, \\
& \text { if }\left|\mu_{i}^{-}\right| \approx 1 \quad \text { then } \quad \operatorname{Re}\left(j \omega \boldsymbol{\phi}_{q} \cdot \boldsymbol{\phi}_{F}\right)<0 .
\end{aligned}
$$

Separating the waves in such a manner, the wave basis can be rewritten as::

$$
\boldsymbol{\Psi}=\left[\begin{array}{cc}
\boldsymbol{\phi}_{q}^{+} & \boldsymbol{\phi}_{q}^{-} \\
\boldsymbol{\phi}_{F}^{+} & \boldsymbol{\phi}_{F}^{-}
\end{array}\right]
$$

\subsection{The Diffusion Matrix Model}

The Diffusion Matrix Model [22] considers two periodic waveguides coupled to each other through a junction which is called the coupling element (Figure 2), and evaluates how wave propagates through such a system.

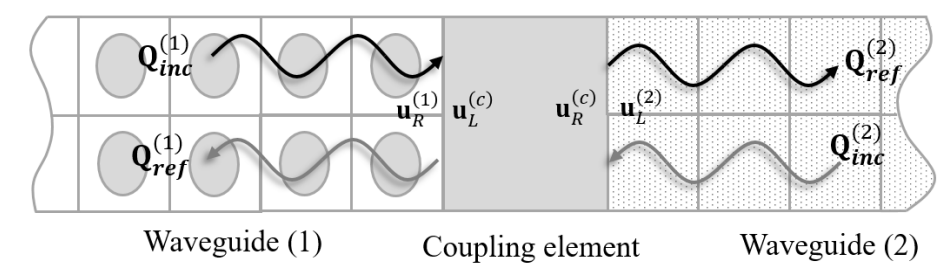

Figure 2: Schematic view of two semi-infinite waveguides coupled through a junction

In the first step, the continuity at the left and right boundary of the coupling element are used to relate its field variables to the variables of each waveguide:

$$
\mathbf{u}_{R}^{(1)}=\mathbf{u}_{L}^{(c)}, \quad \mathbf{u}_{R}^{(c)}=\mathbf{u}_{L}^{(2)}
$$

By substituting the relations above in the coupling element equation of motion, the following equation is obtained in function of the waveguide's field variables:

$$
\mathbf{u}_{L}^{(2)}=\mathbf{S}^{(c)} \mathbf{u}_{R}^{(1)}
$$

where $\mathbf{S}^{(c)}$ is the transfer matrix of the coupling element.

The next step is to expand the field variables at the interfaces using the wave basis of each waveguide:

$$
\mathbf{u}_{L}^{(2)}=\boldsymbol{\Psi}^{(2)}\left[\begin{array}{c}
\mathbf{Q}_{r e f}^{(2)} \\
\mathbf{Q}_{i n c}^{(2)}
\end{array}\right], \quad \mathbf{u}_{R}^{(1)}=\Psi^{(1)}\left[\begin{array}{c}
\mathbf{Q}_{i n c}^{(1)} \\
\mathbf{Q}_{r e f}^{(1)}
\end{array}\right]
$$

where $\mathbf{Q}_{i n c}^{(1)}$ is the amplitude of incident waves at the interface of waveguide (1) and the coupling element, while $\mathbf{Q}_{i n c}^{(2)}$ is the amplitude of incident waves at the waveguide (2) and the coupling element. Moreover, $\mathbf{Q}_{r e f}^{(1)}$ and $\mathbf{Q}_{r e f}^{(2)}$ represent the amplitude of reflected waves at the interface (1) and the amplitude of reflected waves at the interface (2). 
Substituting the expansions above in equation $[13$, we arrive at the coupling equation:

$$
\mathbf{S}^{(c)} \boldsymbol{\Psi}^{(1)}\left[\begin{array}{c}
\mathbf{Q}_{i n c}^{(1)} \\
\mathbf{Q}_{r e f}^{(1)}
\end{array}\right]=\boldsymbol{\Psi}^{(2)}\left[\begin{array}{l}
\mathbf{Q}_{r e f}^{(2)} \\
\mathbf{Q}_{i n c}^{(2)}
\end{array}\right],
$$

which can be reformulated as:

$$
\left(\boldsymbol{\Psi}^{(2)}\right)^{-1} \mathbf{S}^{(c)} \boldsymbol{\Psi}^{(1)}\left[\begin{array}{c}
\mathbf{Q}_{i n c}^{(1)} \\
\mathbf{Q}_{r e f}^{(1)}
\end{array}\right]=\left[\begin{array}{l}
\mathbf{Q}_{r e f}^{(2)} \\
\mathbf{Q}_{i n c}^{(2)}
\end{array}\right] .
$$

The coupling equation (16) relates the amplitude of incident and reflected waves at the interfaces (1) to the amplitude of incident and reflected waves at interface (2).

\subsection{Diffusion based homogenization method}

Diffusion based homogenization method aims at identifying the equivalent coefficients of a partial differential equation chosen a priori using the concept behind CPA, and incorporating DMM to determine the transmission and reflection coefficients numerically instead of using analytic descriptions of the scattering coefficients. Doing so, application can be extended to more complex geometries where computation by analytical means is not allowed due to geometrical complexity. Let's consider a waveguide to be homogenized and call its UC the original UC. Now assume that this waveguide is divided in two parts and an equivalent coupling element is placed in between the two parts (Figure 3), and an incident wave of certain amplitude is arriving at the interface of the waveguide and the equivalent coupling element. The characteristic length of the equivalent coupling element $\mathrm{UC}$ is $L_{c}$, which can differ from the original UC length $L$.

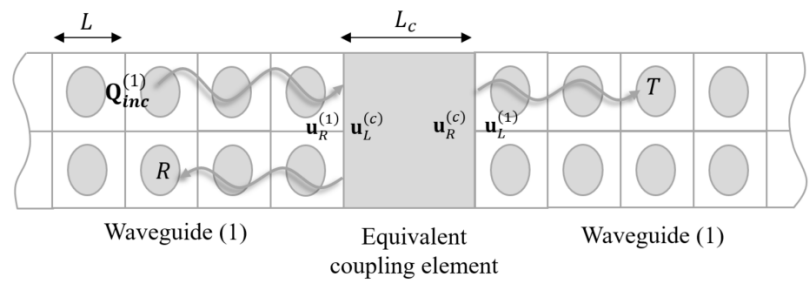

Figure 3: The configuration in which the coupling element is embedded between the targeted waveguides

Since the coupling element is equivalent to the waveguide, the incoming wave should be transmitted completely and have no reflection. Therefore, by minimizing the reflection and maximizing the transmission coefficients at each frequency, the equivalent frequency-dependent material properties can be obtained.

If we assume incident waves of amplitude unity entering our system from left hand side and no incident waves from right hand side, the coupling equation (16) reads:

$$
\boldsymbol{\Psi}^{-1} \mathbf{S}^{(c)} \mathbf{\Psi}\left[\begin{array}{l}
\mathbf{I} \\
\mathbf{R}
\end{array}\right]=\left[\begin{array}{l}
\mathbf{T} \\
\mathbf{0}
\end{array}\right] .
$$


In the equation above, the unknown terms are the transmission $(\mathbf{T})$ and reflection $(\mathbf{R})$ coefficients. Since the wave basis is known and fixed, these terms depend only on the transfer matrix of the coupling element $\left(\mathbf{S}^{(c)}\right)$, which is dependent on the equivalent material properties.

To improve the numerical accuracy of transfer matrix, another method of calculation is proposed in this paper:

$$
\mathbf{S}^{(c)}=\mathbf{A}^{-\mathbf{1}} \mathbf{B} \quad \text { with } \quad \mathbf{A}=\left[\begin{array}{cc}
-\mathbf{D}_{L R}^{(c)} & \mathbf{O}_{n} \\
-\mathbf{D}_{R R}^{(c)} & \mathbf{I}_{n}
\end{array}\right], \text { and } \quad \mathbf{B}=\left[\begin{array}{cc}
\mathbf{D}_{L L}^{(c)} & \mathbf{I}_{n} \\
\mathbf{D}_{R L}^{(c)} & \mathbf{O}_{n}
\end{array}\right]
$$

In equation (17), the transmission and reflection coefficient calculation is highly effected by the well-conditioning of the wave basis. At the same time, accounting for highly evanescent waves results in an ill-conditioned wave basis. Therefore, we propose to keep the necessary waves and to discard the unnecessary ones (highly evanescent waves) using a model order reduction scheme in WFEM [29].

In this paper, part of the calculated wave basis is used as reduction basis to arrive at a reduced set of degrees of freedom, therefore the reduced dynamic stiffness matrix takes the form of:

$$
\mathbf{D}_{\text {red }_{i, j}}=\boldsymbol{\Theta}_{r e d}^{T} \mathbf{D}_{i, j} \boldsymbol{\Theta}_{\text {red }}
$$

where subscripts $i$ and $j$ refer to the left $(L)$ and right $(R)$ sides, and $\boldsymbol{\Theta}_{\text {red }}$ is a $2 n \times n_{\text {red }}$ sub-matrix of the wave basis $\phi_{q}$ with $n_{r e d}$ being the number of modes considered in the reduction basis, which is calculated at each frequency. In the equation above, the reduction wave basis $\left(\boldsymbol{\Theta}_{\text {red }}\right)$ is used as the Galerkin projection of the dynamic stiffness matrix resulting in a reduced dynamic matrix. Readers are referred to [29] for a complete explanation of this model reduction method. It should be noted that in case the wave basis is well-conditioned, this step can be skipped i.e. equating the $\boldsymbol{\Theta}_{\text {red }}$ to the identity matrix.

Afterwards, this reduced dynamic matrix can be used to rewrite the EVP of equation (8) to calculate the reduced wave basis $\left(\boldsymbol{\Psi}_{\text {red }}\right)$. However, considering coupling equation (17), the size of coupling element transfer matrix does not match the truncated wave basis $\left(\boldsymbol{\Psi}_{\text {red }}\right)$. As a result, the coupling element degrees of freedom need to be truncated with the same reduction basis as well, which results in a reduced coupling equation with a similar format as the reduced DMM given in [30]. The final form of the coupling equation is as follows:

$$
\boldsymbol{\Psi}_{r e d}^{-1}\left[\begin{array}{ll}
\mathbf{D}_{r e d_{L R}}^{(c)} & \mathbf{O}_{n} \\
\mathbf{D}_{r e d_{R R}}^{(c)} & \mathbf{I}_{n}
\end{array}\right]^{-1}\left[\begin{array}{cc}
-\mathbf{D}_{r e d_{L L}}^{(c)} & \mathbf{I}_{n} \\
-\mathbf{D}_{r e d_{R L}}^{(c)} & \mathbf{O}_{n}
\end{array}\right] \boldsymbol{\Psi}_{r e d}\left[\begin{array}{l}
\mathbf{I} \\
\mathbf{R}
\end{array}\right]=\left[\begin{array}{l}
\mathbf{T} \\
\mathbf{0}
\end{array}\right]
$$

At last, the target function can be defined such that the transmission coefficients imitate the phase shift across the original UC, while the reflection coefficients are minimized:

$$
f(p)=\left\|\mathbf{P}_{\text {out }}^{\mathbf{T}} \mathbf{T} \mathbf{P}_{\text {in }}-\mathbf{P}_{\text {out }}^{\mathbf{T}} \mathbf{T}_{\mathbf{0}} \mathbf{P}_{\text {in }}\right\|^{2}+\left\|\mathbf{P}_{\text {out }}^{\mathbf{T}} \mathbf{R} \mathbf{P}_{\text {in }}\right\|^{2}
$$

where $\|(\bullet)\|$ is the 2-norm of $(\bullet)$. In this equation, three matrices are introduced. The first one $\left(\mathbf{P}_{\text {in }}\right)$ selects the type of waves to excite. The second one $\left(\mathbf{P}_{\text {out }}\right)$ similarly defines for which waves our material properties will be optimized. 
The effect and description of $\mathbf{P}_{\text {in }}$ and $\mathbf{P}_{\text {out }}$ is explained in more details in section 3.2.2. The last matrix $\left(\mathbf{T}_{\mathbf{0}}=\left(\boldsymbol{\mu}^{+}\right)^{\frac{L_{c}}{L}}\right)$ is the amplitude of transmitted waves, which is assumed to be the same as the incident waves amplitude, considering the phase shift across the coupling element unit cell.

The equation above raises the question whether the choice of coupling element length $\left(L_{c}\right)$ could be done in an arbitrary manner or not. One can argue that in an ideal numerical example such as a homogenized waveguide, the wave-based diagonalization done in equation would be perfect and since the transfer matrix of a structure with $n$ elements is simply the product of $n$ transfer matrices, consequently the mismatch between $L_{c}$ and $L$ would not have an effect on the method. On the other hand, for non-ideal cases where multi-modal interaction and wave conversions can happen, the diagonalization would not be perfect, and would constrain the coupling element to have the same size as the waveguide. Moreover, it is known that considering large elements in WFEM causes numerical problems [11]. Therefore, the coupling element should be discretized in smaller elements, which is not computationally efficient. However, the wave filtering/selection introduced above allows us to have a near perfect diagonalization, such that it would be sufficient for $L_{c}$ to have the same size as the waveguide mesh at the interface. This constraint can be removed if the waveguide is directly coupled to an analytical model. This can be achieved on the condition that kinematic equations are known and the coupling of incompatible interfaces is ensured [22].

\section{Results and discussion}

In this section the diffusion based homogenization method is applied on four case studies. Firstly, a 1D beam is chosen as a proof of concept. The second example is selected to evaluate the capability of the method in capturing complex dynamic behavior, for instance stopband behavior. Therefore, a periodic beam with added tuned vibration absorbers (TVAs) is considered. In this example, we highlight the added value and necessity of the wave type selection step of the method. Thirdly, a more complex geometry and more realistic realization of a structure with stopband behavior, a periodic beam with resonator, is evaluated. In this example, FRFs of the original model and the homogenized model in term of finite and semi-infite beams are calculated and compared to each other. Finally, a cantilever beam with box cross-section and resonators attached to its outer boundary is considered to evaluate the efficiency of the method in a more complex structure. The comparison is done in term of FRFs.

An unconstrained gradient free optimization in MATLAB and Optimization Toolbox Release 2016b (fminsearch function) is used to derive the material properties in all the examples.

\subsection{Case study 1:proof of concept}

In this part, a $1 \mathrm{D}$ steel $\left(E=210 \mathrm{GPa}, \rho=7800 \mathrm{~kg} \cdot \mathrm{m}^{-3}, \eta=0.001\right)$ beam is chosen as proof of concept. The UC is consists of 10 2DOFs per node beam elements with the total length $(L)$ of $10 \mathrm{~cm}$, while the coupling element is a beam element of length $L_{c}=1 \mathrm{~cm}$, with random values $\left(E_{0}=210 \mathrm{GPa}, \rho_{0}=100 \mathrm{~kg} \cdot \mathrm{m}^{-3}, \eta_{0}=1\right)$ for the material properties. The optimization parameters are the density $(\rho)$ and the damping $(\eta)$. Moreover, the reduction scheme is 
not used in this example, since there are only two modes propagating in the beam elements. The material properties at all frequencies was retrieved with a maximum relative error of $4.10^{-6}$ for density and $4.10^{-5}$ for damping. The number of iteration per frequency for the optimization to converge is shown in Figure 4

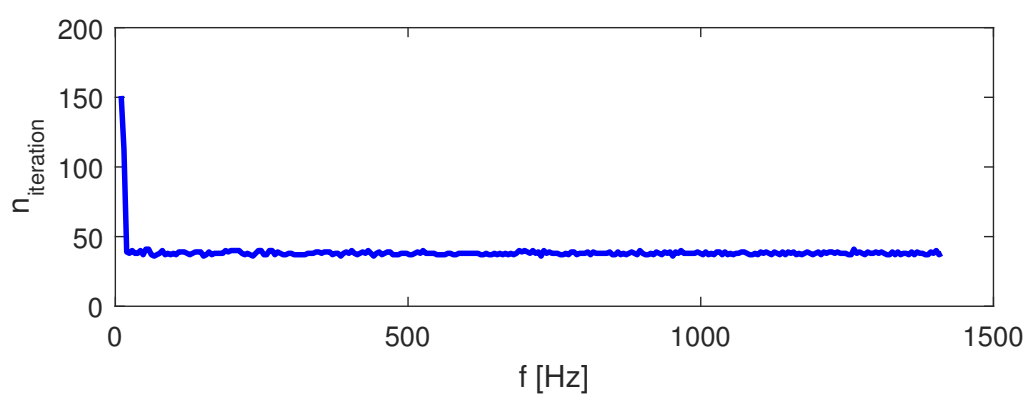

Figure 4: The number of iteration per frequency to optimize the homogenization material properties.

The dispersion curves of the original model and its equivalent one are compared in Figure 5 , which shows excellent agreement as expected.
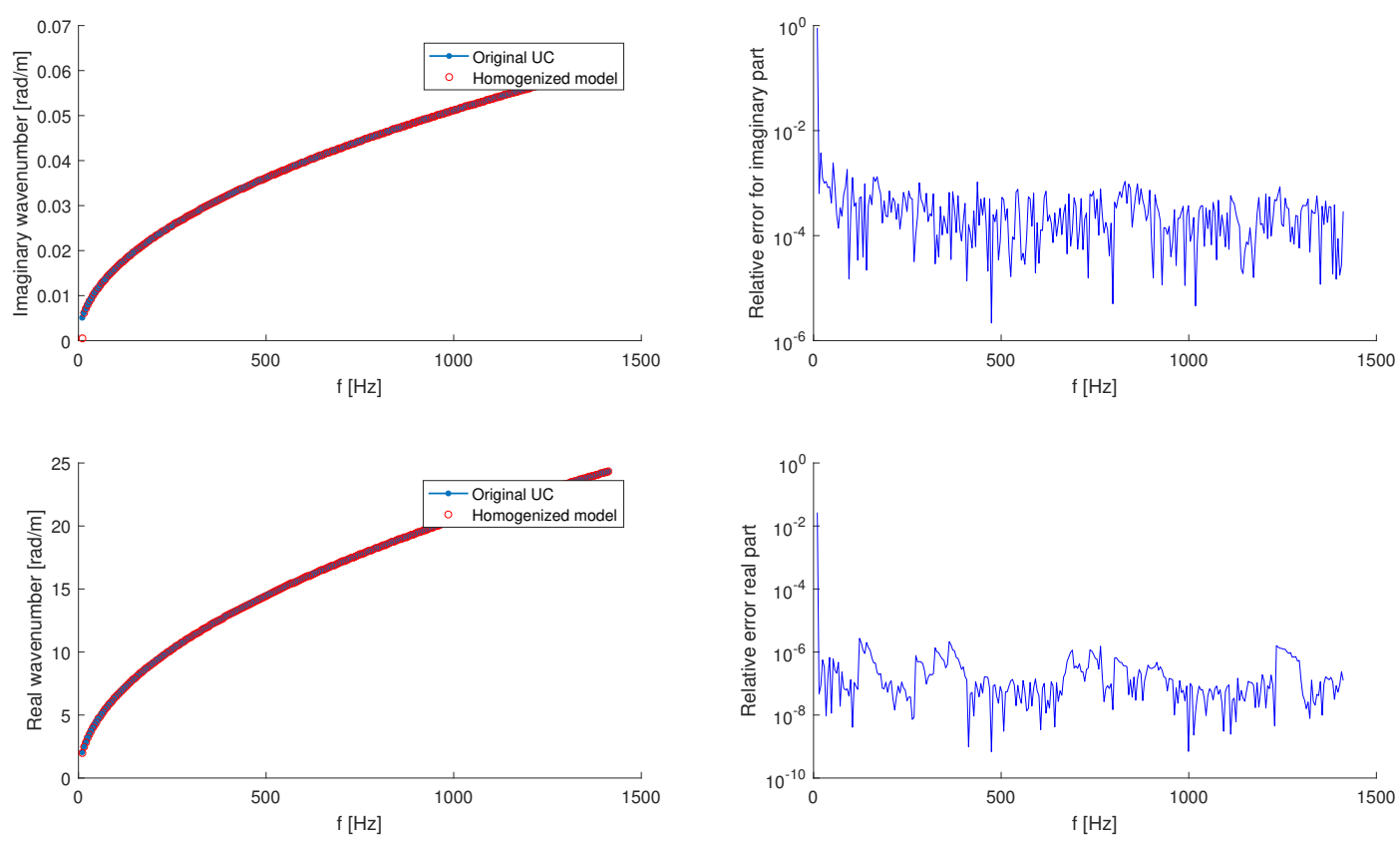

(a)

(b)

Figure 5: Dispersion curve comparison of the 2DOF homogenize beam with its equivalent model (a), and their relative error (b). 


\subsection{Case study 2: Beam with TVAs}

In the last decades, locally resonant metamaterials have gained attention in vibro-acoustic applications. In this context, the out-of-plane bending motion is the most important behavior, since this wave couples to acoustic waves, and causes the structure to radiate noise.

This section is divided in three parts. Firstly, a description of the case to be homogenized is given in detail. Secondly, the effect of the wave type selection in two parts of the homogenization procedure, being wave basis reduction and correction matrices in the target function, is investigated. At last, the equivalent density is evaluated over the frequency range of the problem.

\subsubsection{Problem description}

An aluminum beam with added TVAs is considered in this section. The waveguide and the equivalent coupling element junction in between is shown in Figure 6

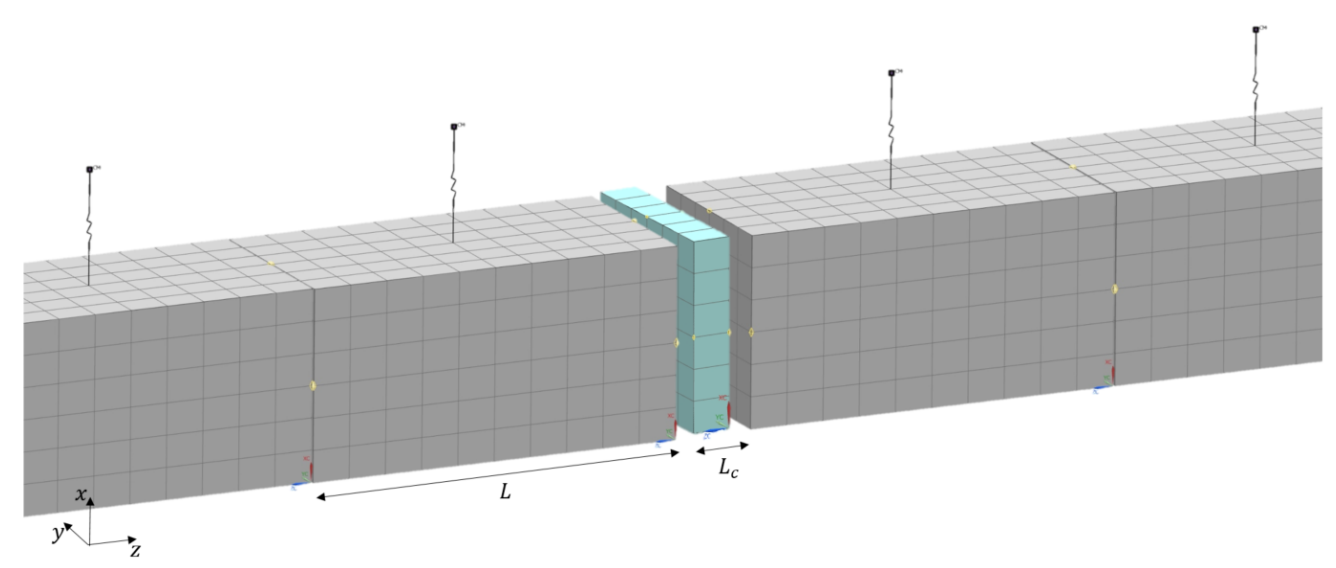

Figure 6: Finite element model of the beam with TVA and the coupling element

The original UC and the coupling element are modeled using the NX 12.0 and are discretized using linear CHEXA8 elements, while the TVAs are modelled using a CELAS1 elements with translation stiffness along $x$-axis. The material properties, dimensions of the unit cell, and characteristics of the TVAs are given in the Tables 1 and 2. Moreover, the optimization variables are the density $(\rho$,$) and the hysteresis damping (\eta)$, because at low-frequencies the dynamic stiffness matrix is mostly effected by the dynamic mass [21].

\begin{tabular}{ccccc} 
Youngs modulus & Poissons ratio & Density & Dimension & Hysteresis damping \\
\hline \hline $68.9 \mathrm{GPa}$ & 0.27 & $2700 \mathrm{~kg} / \mathrm{m}^{3}$ & $25 \times 25 \times 50 \mathrm{~mm}$ & 0.01
\end{tabular}

Table 1: Material properties of the beam and its unit cell dimension 


\begin{tabular}{ccc} 
Added mass & Tuned frequency & Hysteresis damping \\
\hline \hline $0.2 m_{U C}$ & $200 \mathrm{~Hz}$ & 0.001 \\
& \multicolumn{2}{c}{ Table 2: Characteristics of TVAs }
\end{tabular}

\subsubsection{Influence of wave type selection}

Wave type selection is introduced in two parts of the homogenization procedure: (i) in the wave basis reduction part and (ii) via $\mathbf{P}_{\text {in }}$ and $\mathbf{P}_{\text {out }}$ in the target function. Therefore, in the first case the parameters for step (i) are varied while step (ii) is kept fixed, in the second case the parameters of step (ii) are varied while the parameters of step (i) are fixed. As mentioned earlier, to improve the condition number of the wave basis, a reduction method is applied on the original UC. Therefore, a strategy should be defined to chose the number of modes $\left(n_{\text {red }}\right)$ used as reduction basis. In this paper, we introduced a propagation ratio, which is the ratio between the real part of the wavenumber and imaginary part of wavenumber i.e. $\alpha=\frac{i m a g(\log (\mu))}{\operatorname{real}(\log (\mu))}$, to chose the reduction basis. As a result, the first $n_{\text {red }}$ number of modes with highest value of propagation ratio is chosen as the reduction basis.

The condition numbers of the truncated wave basis using reduction bases of different size are shown in Figure 7 . This demonstrates the improvement of the complete wave basis from a singular matrix to a well-conditioned matrix. Besides improving the accuracy of the optimization, the size of the equation to be solved at each iteration is reduced and consequently reduction in computation time.

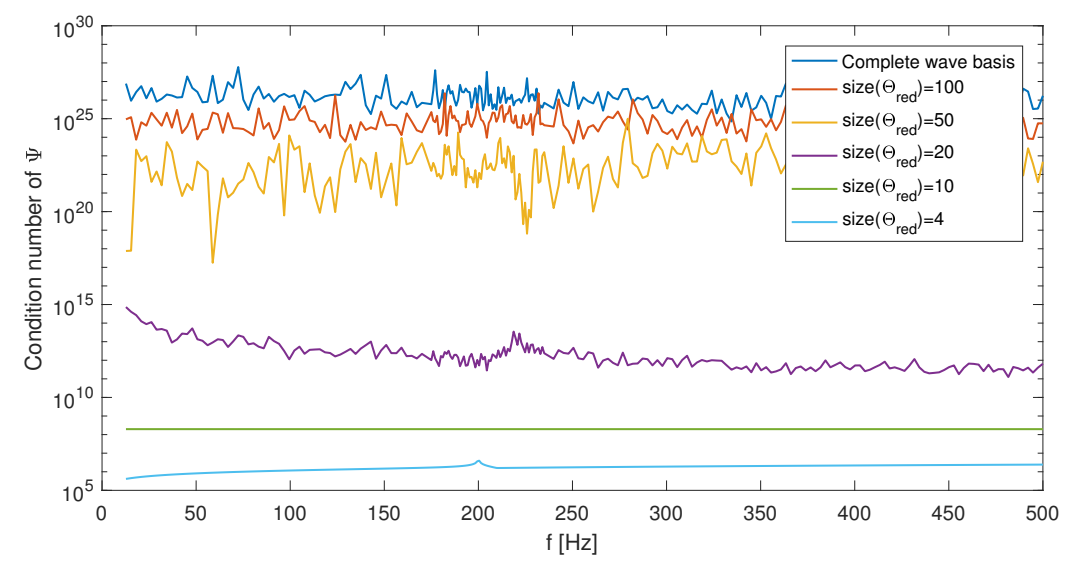

Figure 7: Condition number of the wave basis over frequency for different reduction bases

To further, illustrate the necessity of this step, the bending wave dispersion curves of the equivalent model using different reduced wave bases are calculated and compared to the one of the original UC (Figure 8). Additionally, for reduction bases of the first 4 and 10 modes, the relative error of both real and imaginary part of the wavenumbers between the original UC and the equivalent model is shown in Figure 9 . 

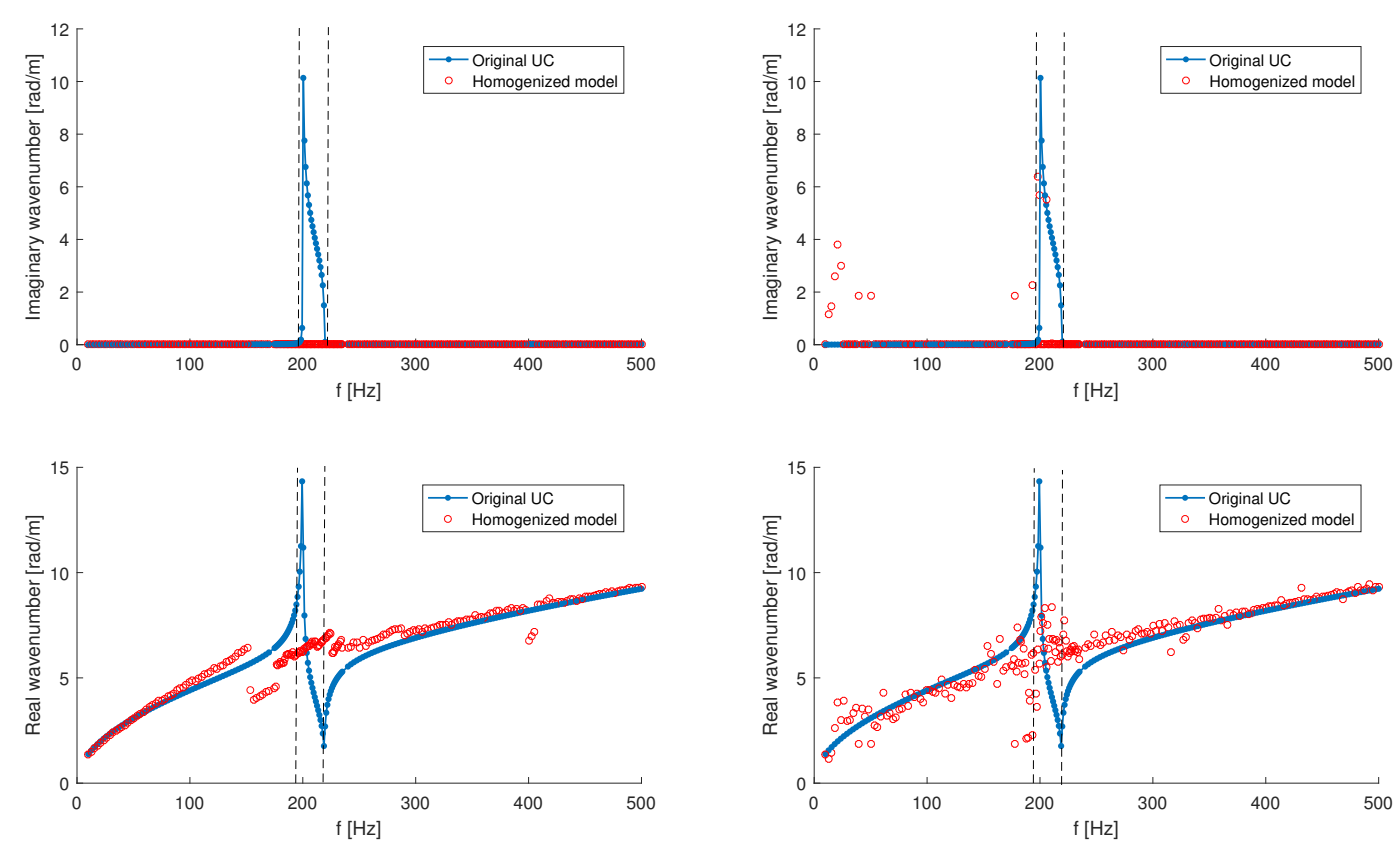

(a)

(b)
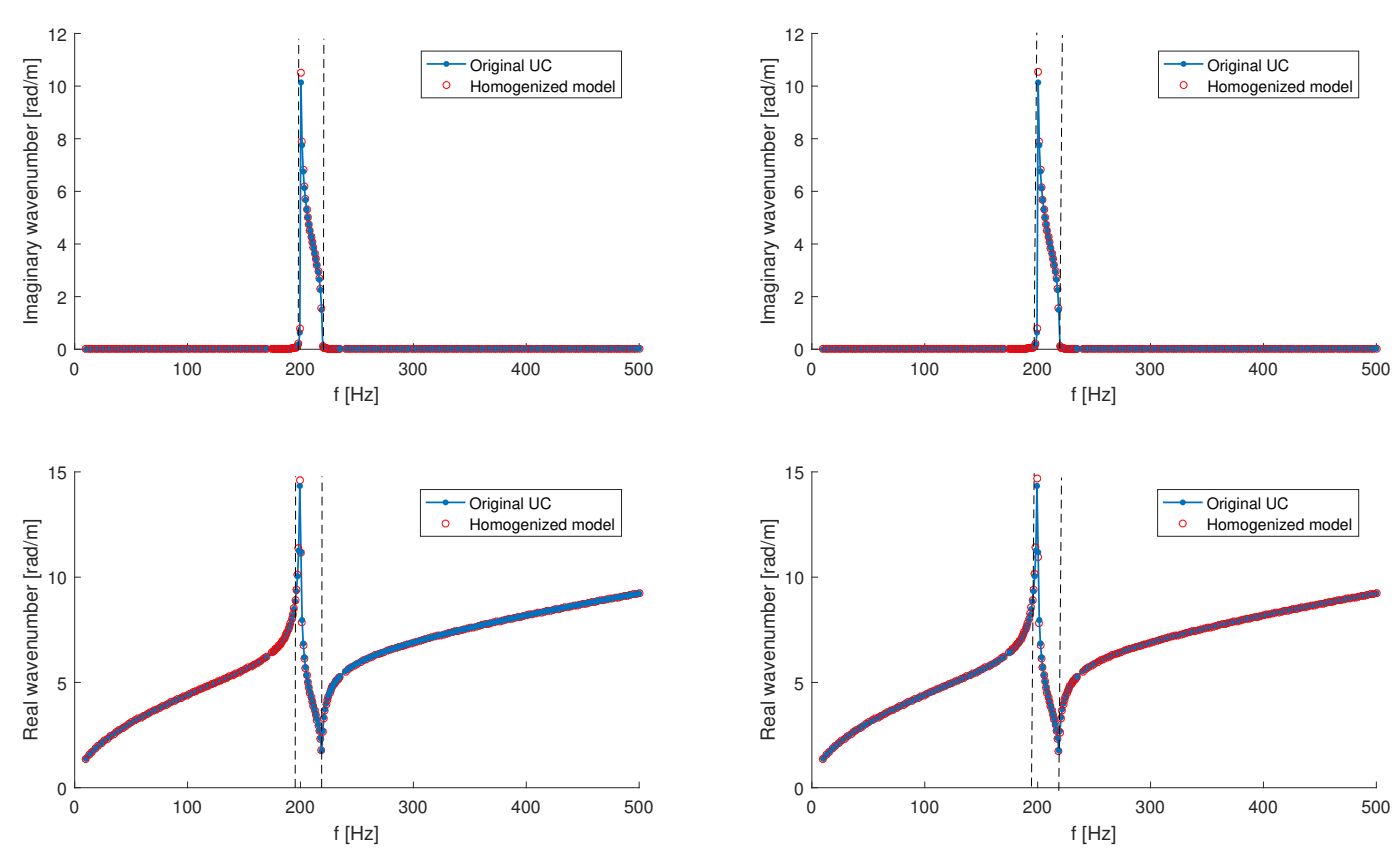

(c)

(d)

Figure 8: Dispersion curve (bending wave) comparison between the beam with TVAs and its equivalent model obtained using the first (a) 100 modes, (b) 50 modes, (c) 10 modes, (d) 4 modes of the wave basis as the reduction basis 

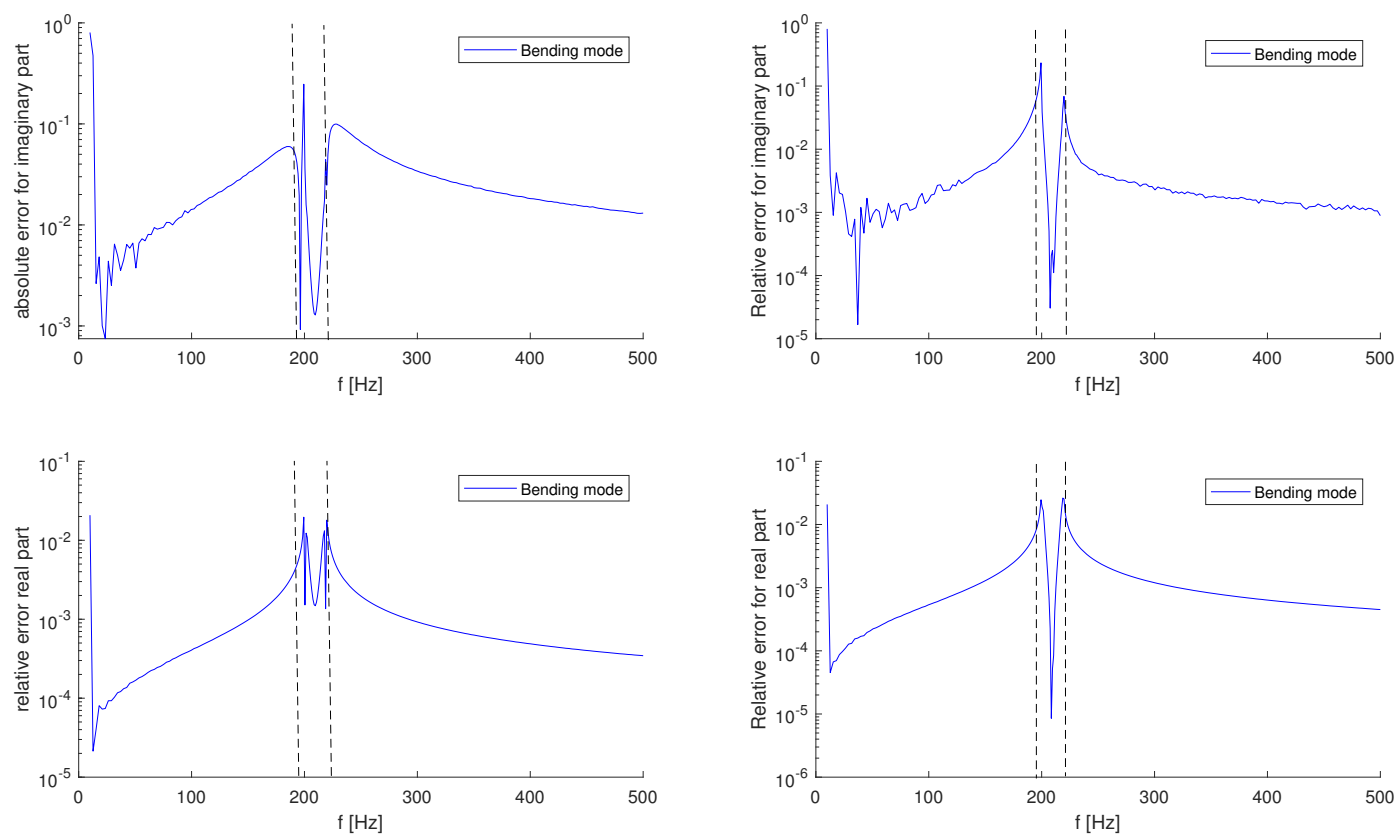

(a)

(b)

Figure 9: Relative error between the bending wave dispersion curves of the original UC and its equivalent model obtained using the first (a) 10 modes, (b) 4 modes of the the wave basis as the reduction basis

Figure $8(\mathrm{~d})$ shows a good agreement for both imaginary and real part of the wavenumber with a mean value of $0.1 \%$ error for the real part and mean value of $2 \%$ for the imaginary one, and slightly higher error in the stopband (zone between the dashed vertical lines in Figure 8 and 9]. It is expected that the higher error in the stopband is caused by the fact that higher order evanescent waves contribute to the behavior in this frequency zone which are not accounted for in this method due to the condition number of the wave basis. Next, we evaluate the effect of $\mathbf{P}_{\text {in }}$ and $\mathbf{P}_{\text {out }}$ on the optimization by comparing two cases. In the first case, all wave types are excited and the equivalent material properties are optimized for all the wave types $\left(\mathbf{P}_{\text {in }}=\mathbf{I}\right.$ and $\left.\mathbf{P}_{\text {out }}=\mathbf{I}\right)$. In the second case, only the wave type of our interest (bending wave along $y$-axis) is excited and the scattering coefficients of the same wave are optimized, which means only diagonal indices related to the desired bending mode are non-zero in the $\mathbf{P}_{\text {in }}$ and $\mathbf{P}_{\text {out }}$ matrices. The dispersion curves of the bending wave of the equivalent model of these two case are compared to dispersion curves of the original UC in Figure 10. It is apparent that exciting and optimizing the material properties for all wave type leads to inaccurate results due to the fact that original UC is replaced by an isotropic medium with inherent fixed relationships between wave types (shear, longitudinal, and two bending modes) when the structure exhibits complex dynamic behavior (resonance behavior) for only one of the wave types. In other word, considering behaviors that the homogenized model (isotropic medium) cannot capture inherently, degrades the accuracy. Therefore, for cases 
exhibiting more than one dominant behavior such as muti-modal wave propagation, and wave conversion, a more enriched homogenized model should be considered.
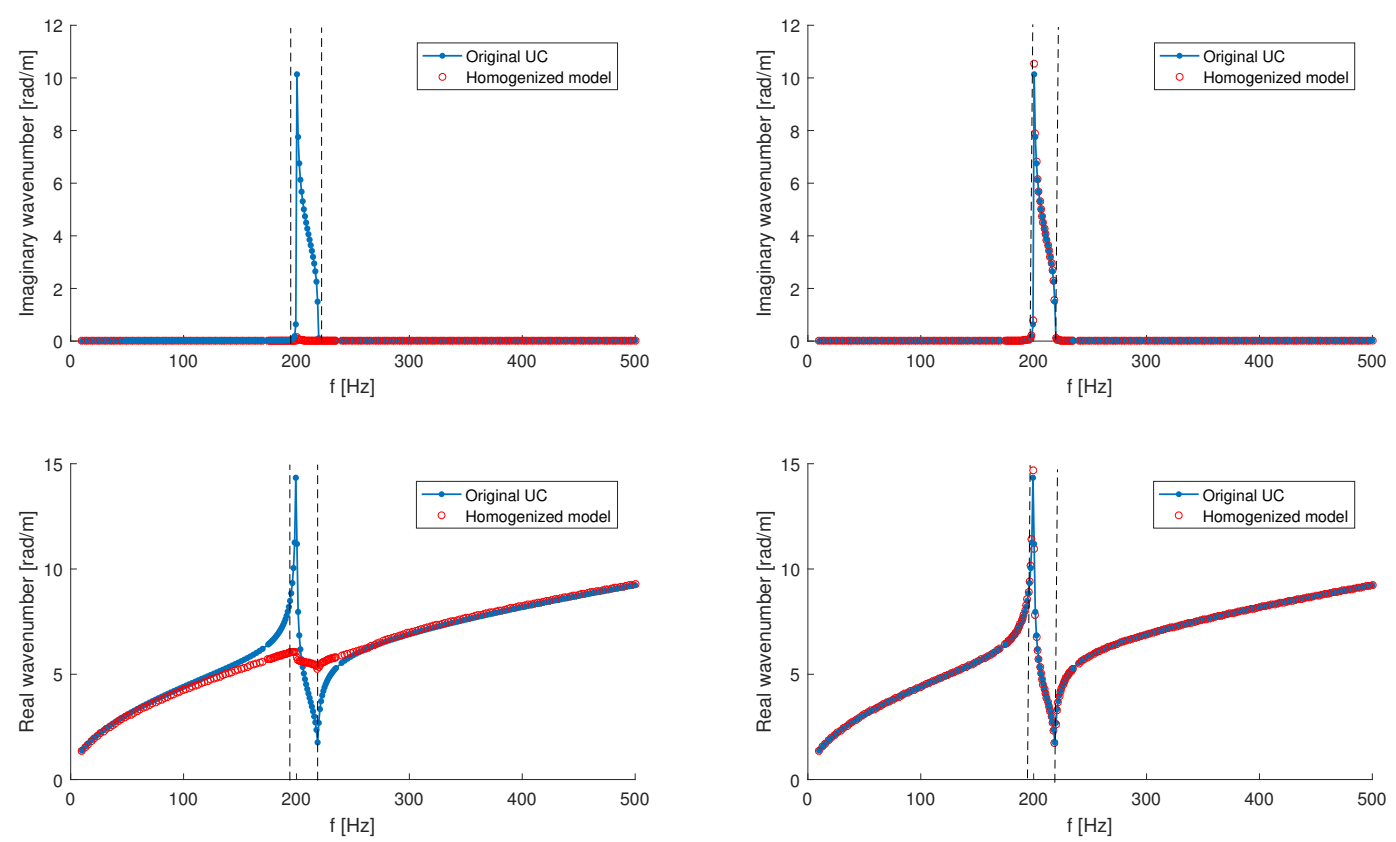

(a)

(b)

Figure 10: Dispersion curve comparison between the beam with TVAs and its equivalent model obtained exciting and optimizing all wave types (left), and exciting and optimizing only bending wave (right)

\subsubsection{Equivalent density}

In this section the physical meaning behind the converged values of the density is explained. The comparison is between the equivalent density and the dynamic density [21] of the beam (not the nominal density of the beam), because the equivalent density is derived from a dynamic homogenization approach and not a static one. The dynamic density/mass can be calculated analytically for locally resonant metamaterials by considering the structure as a lumped 2DOF system and then replacing the 2DOF system with a 1DOF system with dynamic density/mass, which is formulated as follows [21]:

$$
\widetilde{m}=m_{1}+m_{2}\left[\frac{\frac{2 i \eta_{2} \omega}{\omega_{2}}+1}{1+\frac{2 i \eta_{2} \omega}{\omega_{2}}-\left(\frac{\omega}{\omega_{2}}\right)^{2}}\right],
$$

where $\widetilde{m}$ is the dynamic mass/density, $m_{1}$, and $m_{2}$ are the host structure and the TVA mass/density. $\eta_{2}$ is the damping ratio of the TVA, and $\omega_{2}$ is the natural frequency of the TVA. 


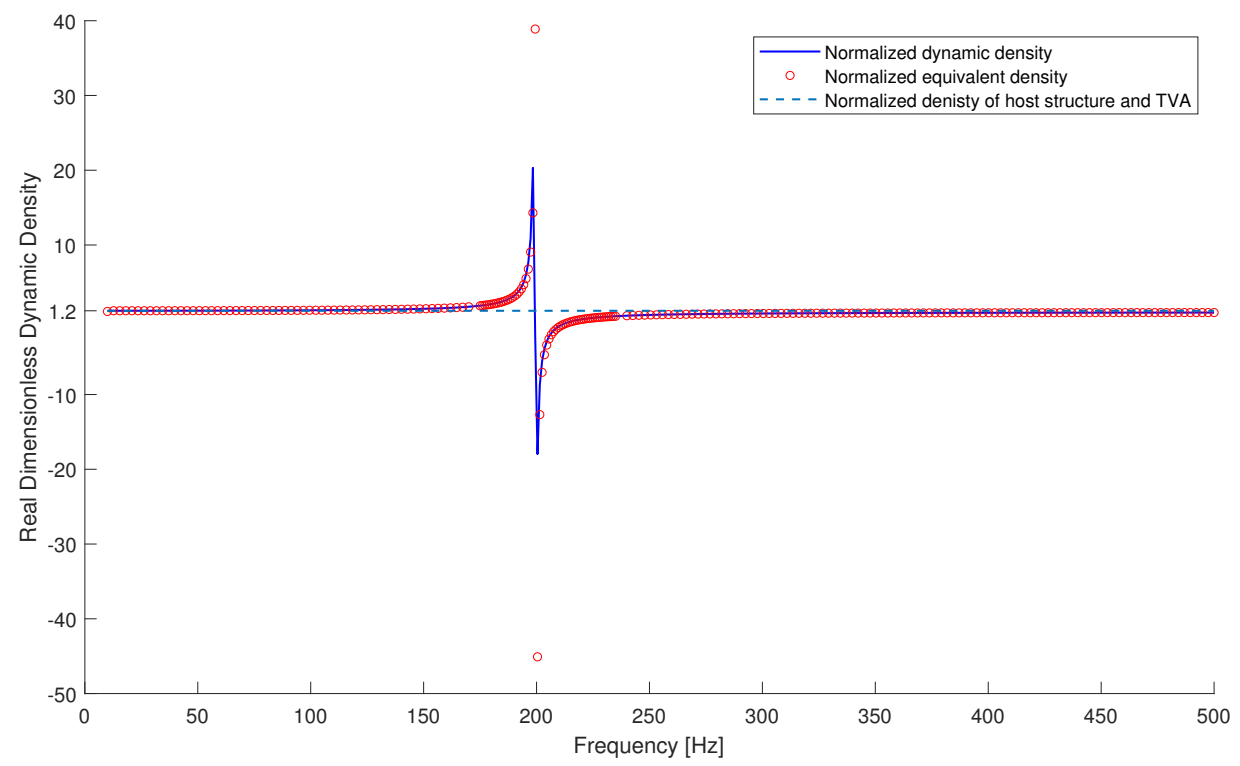

Figure 11: Dynamic density and the converged value of density $n$ the homogenization

In Figure 11, the dynamic density of the structure is compared to the equivalent density. All values in Figure 11 are divided by static density of the host structure $\left(\rho=2700 \mathrm{~kg} / \mathrm{m}^{3}\right)$. To have a better view, the summed density of the host structure and the TVA $\left(\rho_{\text {tot }}=1.2 \rho\right)$ is plotted as well. At low frequencies the dynamic density is equal to the total static density of the structure $\left(\rho_{t o t}=1.2 \rho\right)$. Going towards the resonance frequency of the TVA, the dynamic density tends to infinity. After the resonance frequency, the TVA and the host structure move out-of-phase (anti-resonance behavior) resulting in negative dynamic density. Afterwards, at higher frequencies the dynamic density evolves to the static density of the host structure $(\rho)$, since the coefficient multiplied by $m_{2}$ (Eq. (22) ) tends to zero.

Looking more closely at the Figure 11, two extremes are noticed which do not fit well with the dynamic density, which can be explained by the fact that the definition of the dynamic density given in [31] already takes into account the effect of damping in the resonator, whereas in the model of this paper, damping is added separately through the equivalent damping parameter. Therefore, causing the extremes of dynamic density to be pushed down. 


\subsection{Case study 3: Beam with Resonators}

In this section, the advantage of the method is highlighted for the case of a realistic structure and the dynamic response of the structure is considered due to force excitation. It is shown that the equivalent model can be used in infinite analysis as well as finite analysis. Therefore, this section is divided in three parts. The first one introduces the case study. The second one compares infinite analysis of the equivalent model to the one of original structure. The third one evaluates the FRF of the original structure in comparison to the one of the equivalent model.

\subsubsection{Problem description}

An aluminum beam with resonators attached at the top is considered, which is shown in Figure 12 together with the equivalent coupling element. The beam, resonator, and the coupling element are modeled using quadratic CHEXA20 elements in NX 12.0 software. The dimension of the original UC is $25 \times 25 \times 50 \mathrm{~mm}$. Its FE representation consists of 13104 degrees of freedom (DOFs). The resonator is tuned to $522 \mathrm{~Hz}$ and its material is Polymethyl methacrylate (PMMA). Material properties are summarized in Table 3.

The optimization variables in this case are the density $(\rho)$ and the hysteresis damping $(\eta)$.

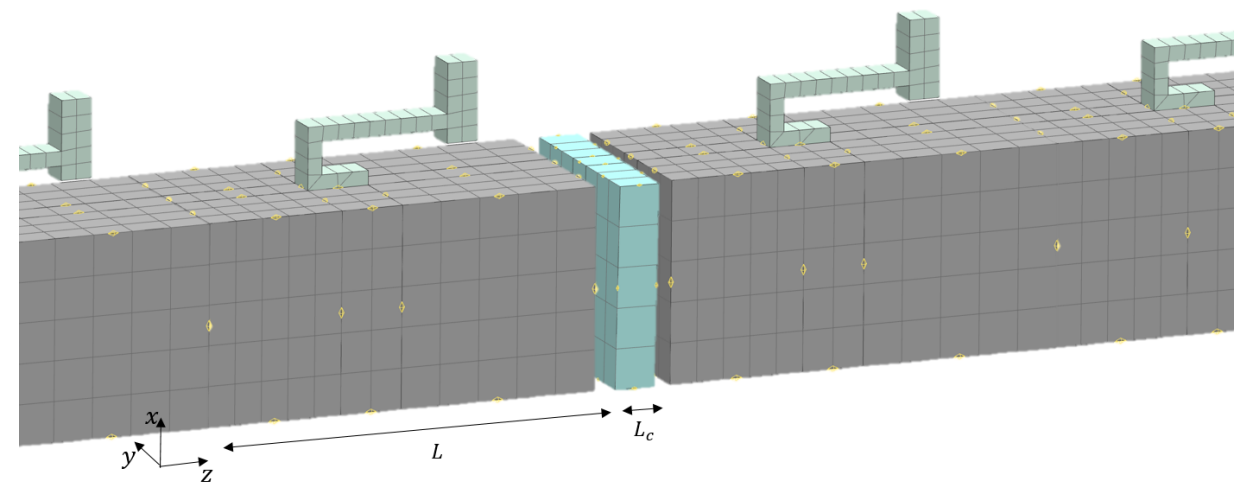

Figure 12: Finite element model of the beam with resonators and the coupling element

\begin{tabular}{cccc} 
Youngs modulus & Poissons ratio & Density & Hysteresis damping \\
\hline \hline $4.85 \mathrm{GPa}$ & 0.3 & $1188.38 \mathrm{~kg} / \mathrm{m}^{3}$ & 0.001 \\
& \multicolumn{2}{c}{ Table 3: Properties of PMMA }
\end{tabular}

\subsubsection{Infinite structure analysis}

Firstly, the wave basis of the original UC is calculated and truncated using the first 4 propagative modes as the reduction basis e.g. $n_{\text {red }}=4$. Secondly, in the optimization step the bending mode around the $y$-axis is excited and the homogenization parameters are optimized for the same mode. The average optimization time is $7.1 s$ per frequency. Finally, the derived equivalent material properties are plugged into the analytical dispersion relation (equation 23 for 
an Euler-Bernoulli bean to calculate the dispersion curve of the equivalent model. In Figure 13, the semi-analytical dispersion curve of the equivalent model is compared to the dispersion curve of the original UC, calculated using the WFEM.

$$
k_{\text {analytical }}=\sqrt[4]{\omega^{2} m /(E I)} .
$$

The dispersion curve comparison shows a good agreement between the original model and its equivalent model.
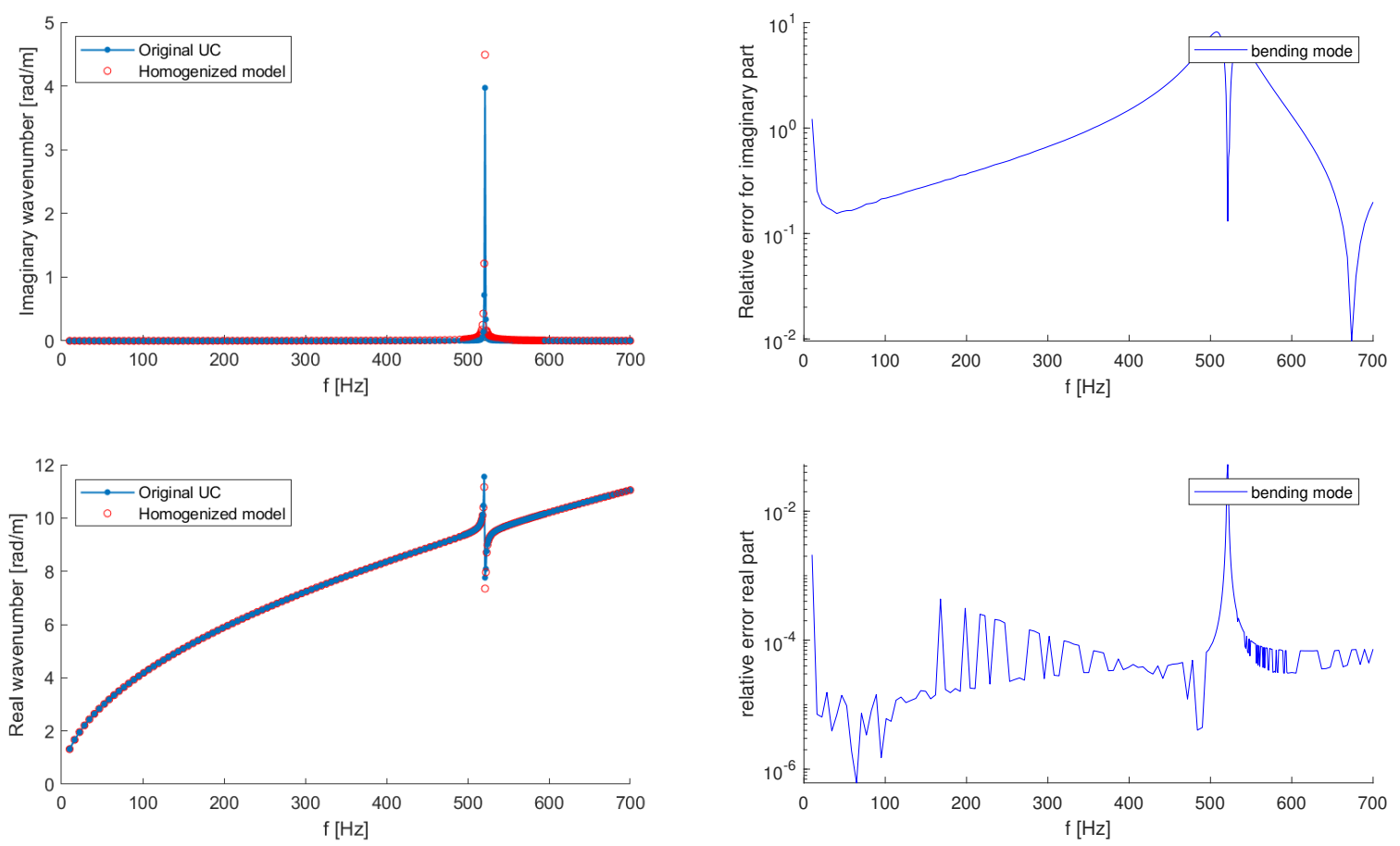

(a)

(b)

Figure 13: Dispersion curve comparison between the beam with resonators and its equivalent semi-analytical model (a) and the relative error between the two (b)

\subsubsection{Frequency response function}

In this part, the FRFs of the equivalent model and the original structure are compared to each other for semi-infinite and finite beams.

\subsubsection{Semi-infinite beam}

The FRF of a semi-infinite beam constructed with the original UC and excited at its free end in the out-of-plane direction is calculated using WFEM [29] and is compared to the FRF of a semi-infinite beam built with the equivalent 
model in Figure 14 In this figure, the response locations are 3,6,10, and $20(N)$ unit cells from the free edge. Since the length of the original UC $(L)$ is different from the homogenized UC $\left(L_{c}\right)$, the number of homogenized UCs $\left(N_{c}\right)$ to have the same response location is calculated by scaling $N$ i.e. $N_{c}=\frac{L}{L_{c}} N$. Moreover, the relative error between the FRF of the original model and the homogenized one is shown in Figure 15.
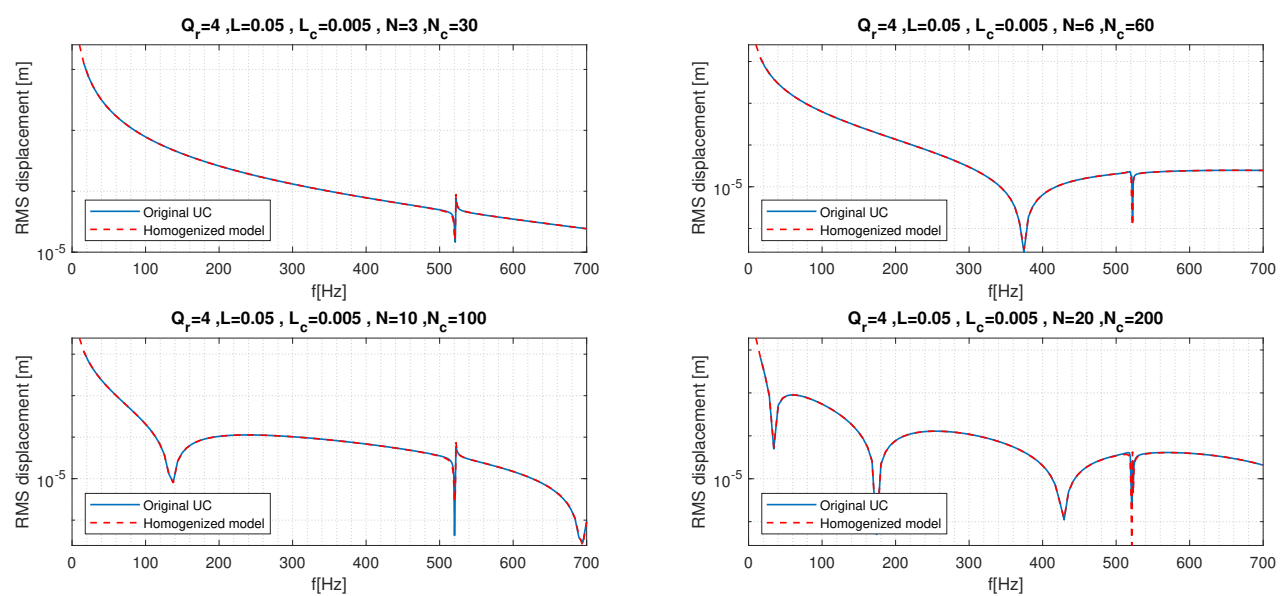

Figure 14: FRF of the semi-infinite beam compared to its homogenized model at different response location
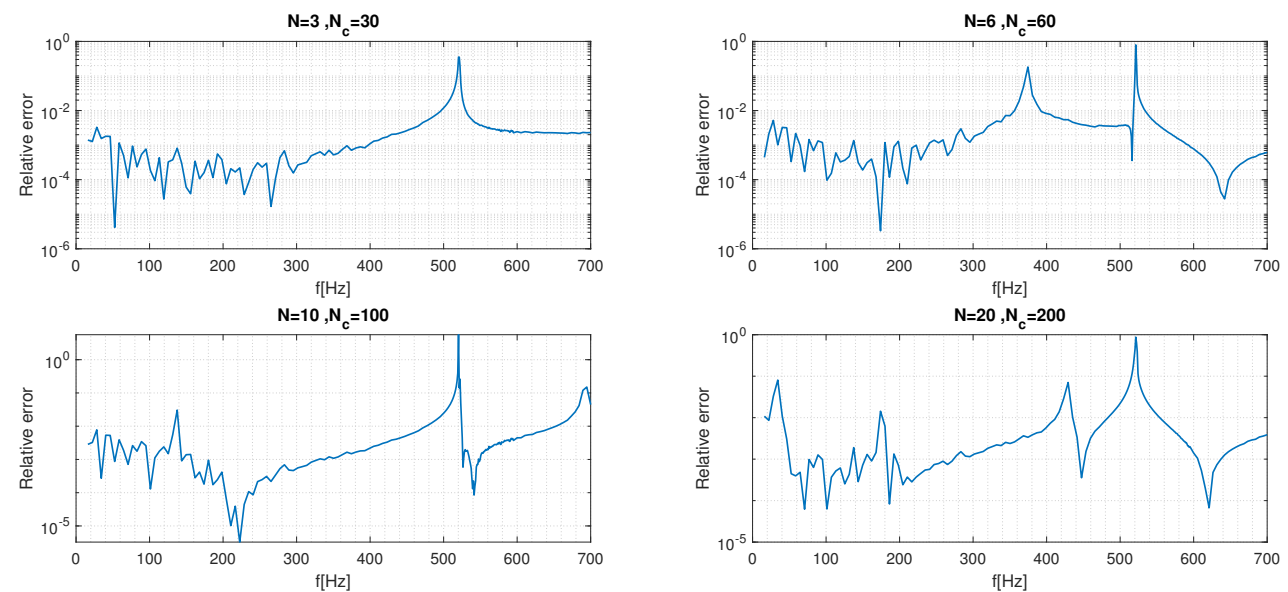

Figure 15: Relative error between the FRF of the semi-infinite beam and its homogenized model at different response location

The resonance frequency of the resonator at $522 \mathrm{~Hz}$ is noticeable in both models, while some dips appear at both low and high frequencies when the response location is taken further away. These dips refer to nodal points. 


\subsubsection{Finite beam}

A $1.15 m$ cantilever beam constructed with the original UC, and excited at its free end in the $x$ direction with a point source is considered. Its FRF is calculated using COMSOL Multiphysics 5.3a. Moreover, the exact response solution [32] of an Euler-Bernoulli cantilever beam excited with harmonic point load at its free end using the identified equivalent material properties is calculated. The comparison between the two responses is shown in Figure 16 , showing a good agreement. It can be seen that the homogenized model overestimates the damping around the bandgap limits (the vertical lines in Figure 16, while underestimating the damping at the resonance frequency of the resonators $(522 \mathrm{~Hz})$ due to smoothness of the target function in the bandgap.

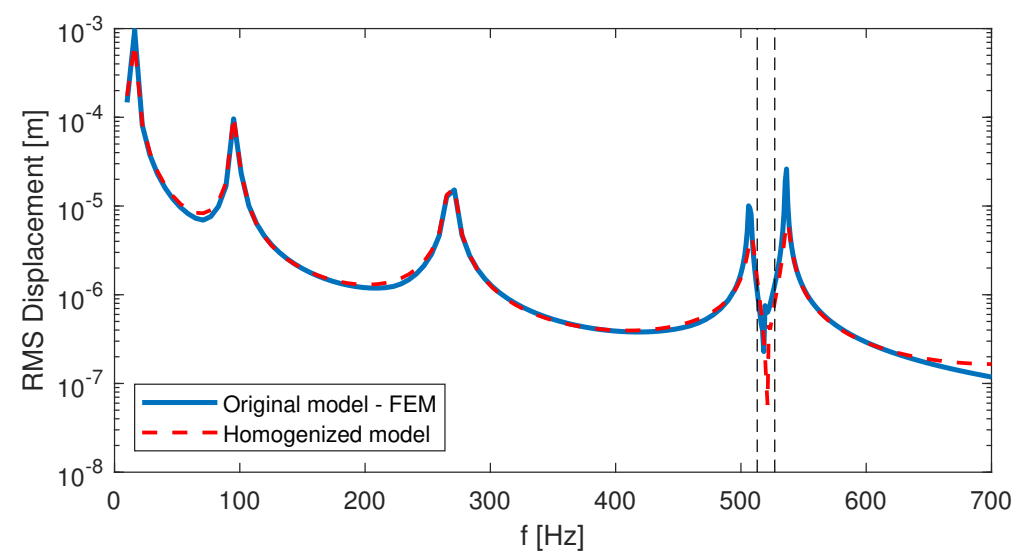

Figure 16: FRF of the RMS displacement of a cantilever beam excited at its free end calculated using FEM (blue line), and the exact solution of an Euler-Bernoulli beam with equivalent material properties (red dashed line).

\subsection{Case study 4: Beam with box cross-section}

As a final example, a more complex and computationally demanding case is studied to evaluate the efficiency of the method. A box cross-section is made by repeating and rotating the UC considered in case study 3 in the $x-y$ planes, see Figure 17. The square box has an outer dimension of $125 \mathrm{~mm}$ and an inner dimension of $75 \mathrm{~mm}$. A cantilever beam of $1.1 \mathrm{~m}$ is constructed by repeating the cell of Figure 17 along the $z$-axis. The beam is excited with a harmonic point force at its free end.

The RMS dynamic response of the beam in terms of displacement is calculated using WFEM combined with the MOR technique presented in [13]. This result is compared to the analytical solution of a Timoshenko beam theory [32] with a box cross-section and the equivalent material properties (damping and density) retrieved from the previous case, see Figure 18. It can be seen that while the homogenized model response is in good agreement with the original model response, it overestimates the damping before and after the bandgap limits. This was expected since the equivalent material properties used in this case are based on the ones of Case study 3, in which damping overestimation was observed. 


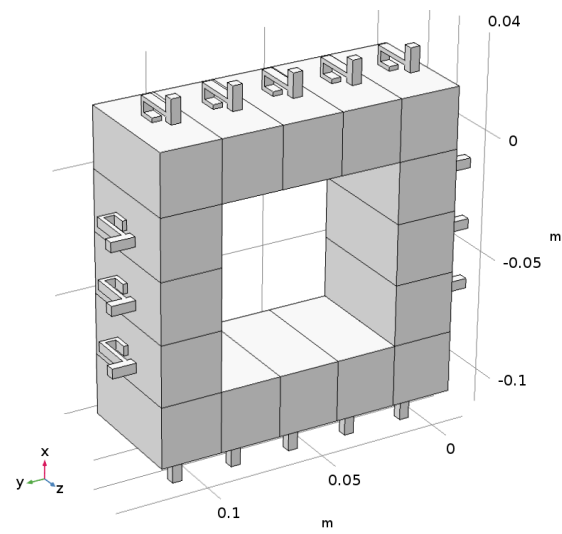

Figure 17: Box cross-section of the beam constructed using the UC of case 3.

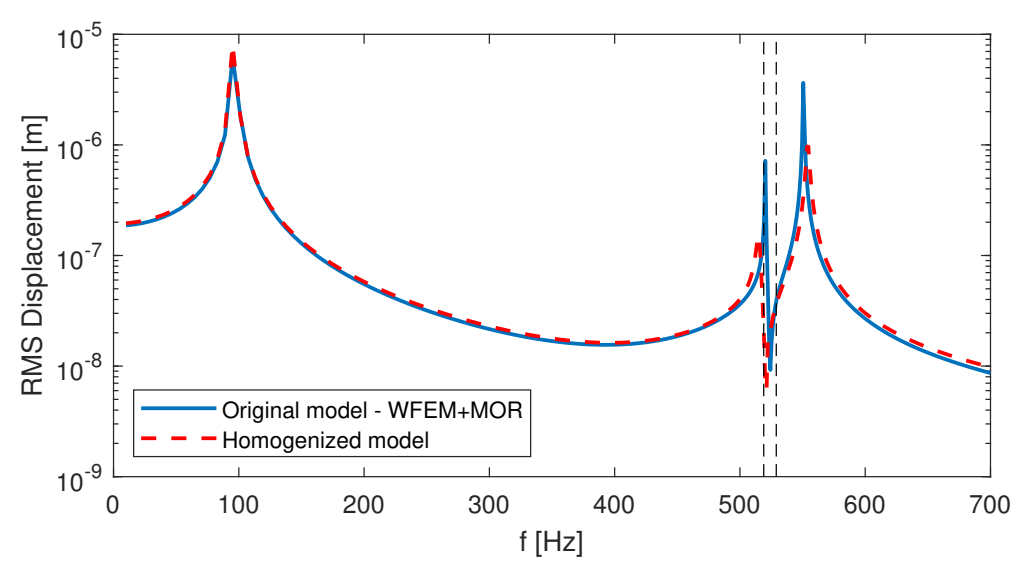

Figure 18: FRF of the RMS displacement of the cantilever beam with box cross-section excited at its free end calculated using WFEM combined with MOR (blue line), and the exact solution of an Timoshenko beam with equivalent material properties (red dashed line).

\section{Conclusion}

This paper presents a dynamic homogenization approach for 1D periodic waveguides, exploiting the WFE framework within the CPA context, where the equivalent model, that is chosen a pririo, is embedded between two identical waveguides and the equivalent properties are derived by optimizing the scattering coefficients. It is shown that using the Diffusion Matrix Model to calculate the scattering coefficients numerically, enables the proposed method to handle structures with more complex geometry and more complex dynamic behavior such as stopband behavior, which provides an advantage with respect to other available dynamic homogenization methods in literature. In this paper an Euler-Benoulli beam theory and/or Timoshenko beam theory is chosen as the equivalent model since it leads to classical material properties (though with unconventional values) and identical sections for the homogenized coupling element and the original model, which simplifies the implementation of the method. However, it is possible to couple the waveguide directly to any analytical model for wave propagation (Euler Bernoulli, Timoshenko, and more 
enriched beam theories) given that the corresponding tensors/kinematic equations of the cross-section are known and that access to the FEM package allows to implement the coupling correctly. It should be noted that the ability of the method to deal with multimodal propagation is predicated on the analytical model used for the homogenization. The more complex the partial differential equation, the more behavior can be captured. In other words, the method is used to identify the coefficient of the partial differential equations when geometry is too complex to allow their computation by analytical means. Therefore, the coefficients can be identified via the optimization problem as long as the general form of the equation is known. In this paper a beam with resonator inclusions and a metamaterial duct are considered to show the ability of the method to capture complex dynamic behavior. Since an Euler-Bernoulli/Timoshenko beam was chosen as an equivalent model, a wave type selection scheme is proposed to filter out the behaviors that the analytical model inherently cannot capture to improve the accuracy of the method. It was shown that this simple model is able to represent the original model with good accuracy in both unit cell analysis and frequency response analysis.

\section{Acknowledgements}

The European Commission is gratefully acknowledged for their support of the VIPER research project (GA675441). The Research Fund KU Leuven is gratefully acknowledged for its support. The research of E. Deckers is funded by a grant from the Research Foundation Flanders (FWO).

[1] Spadoni, A., Ruzzene, M., Gonella, S., Scarpa, F.. Phononic properties of hexagonal chiral lattices. Wave Motion 2009;46(7):435-450.

[2] Khelif, A., Aoubiza, B., Mohammadi, S., Adibi, A., Laude, V.. Complete band gaps in two-dimensional phononic crystal slabs. Physical Review E 2006;74(4):046610.

[3] Hussein, M.I., Leamy, M.J., Ruzzene, M.. Dynamics of phononic materials and structures: Historical origins, recent progress, and future outlook. Applied Mechanics Reviews 2014;66(4):040802.

[4] Claeys, C., Deckers, E., Pluymers, B., Desmet, W.. A lightweight vibro-acoustic metamaterial demonstrator: Numerical and experimental investigation. Mech Syst Signal Process 2016;70-71:853-880.

[5] Zhou, X., Liu, X., Hu, G.. Elastic metamaterials with local resonances: an overview. Theoretical and Applied Mechanics Letters 2012;2(4):041001.

[6] Liu, Z., Zhang, X., Mao, Y., Zhu, Y., Yang, Z., Chan, C.T., et al. Locally resonant sonic materials. science 2000;289(5485):1734-1736.

[7] Vasseur, J., Deymier, P., Frantziskonis, G., Hong, G., Djafari-Rouhani, B., Dobrzynski, L.. Experimental evidence for the existence of absolute acoustic band gaps in two-dimensional periodic composite media. Journal of Physics: Condensed Matter 1998;10(27):6051.

[8] Claeys, C.C., Vergote, K., Sas, P., Desmet, W.. On the potential of tuned resonators to obtain low-frequency vibrational stop bands in periodic panels. J Sound Vib 2013;332(6):1418-1436.

[9] De Melo Filho, N., Van Belle, L., Claeys, C., Deckers, E., Desmet, W.. Attenuation prediction in 1D waveguides using locally resonant metamaterials. Proc ISMA 2016 - Int Conf Noise Vib Eng USD2016 - Int Conf Uncertain Struct Dyn 2016;.

[10] Manconi, E., Mace, B.R.. Wave characterization of cylindrical and curved panels using a finite element method. The Journal of the Acoustical Society of America 2009;125(1):154-163.

[11] Waki, Y., Mace, B.R., Brennan, M.J.. Numerical issues concerning the wave and finite element method for free and forced vibrations of waveguides. J Sound Vib 2009;327(1-2):92-108.

[12] Droz, C., Lainé, J.P., Ichchou, M.N., Inquiété, G.. A reduced formulation for the free-wave propagation analysis in composite structures. Compos Struct 2014;113(1):134-144. 
[13] Boukadia, R.F., Droz, C., Ichchou, M.N., Desmet, W.. A Bloch wave reduction scheme for ultrafast band diagram and dynamic response computation in periodic structures 2018;148(May):1-12.

[14] Mencik, J.M.. A model reduction strategy for computing the forced response of elastic waveguides using the wave finite element method. Comput Methods Appl Mech Eng 2012;229-232:68-86.

[15] Andrianov, I.V., Bolshakov, V.I., Danishevs'kyy, V.V., Weichert, D.. Higher order asymptotic homogenization and wave propagation in periodic composite materials. Proc R Soc A Math Phys Eng Sci 2008;464(2093):1181-1201. doi: \bibinfo\{doi\}\{10.1098/rspa.2007.0267\}.

[16] Fossat, P., Boutin, C., Ichchou, M.. Dynamics of periodic ribbed plates with inner resonance: Analytical homogenized model and dispersion features. Int J Solids Struct 2018;152-153:85-103.

[17] Castillero, J., Otero, J., Ramos, R., Bourgeat, A.. Asymptotic homogenization of laminated piezocomposite materials. International Journal of Solids and Structures 1998;35(5-6):527-541.

[18] Boutin, C., Geindreau, C.. Periodic homogenization and consistent estimates of transport parameters through sphere and polyhedron packings in the whole porosity range. Physical review E 2010;82(3):036313.

[19] Willis, J.R.. Effective constitutive relations for waves in composites and metamaterials. Proc R Soc A Math Phys Eng Sci 2011;467(2131):1865-1879.

[20] Wu, Y., Lai, Y., Zhang, Z.Q.. Effective medium theory for elastic metamaterials in two dimensions. Phys Rev B 2007;76(20):205313.

[21] de Melo Filho, N., Belle, L.V., Claeys, C., Deckers, E., Desmet, W.. Dynamic mass based sound transmission loss prediction of vibro-acoustic metamaterial double panels applied to the mass-air-mass resonance. Journal of Sound and Vibration 2019;442:28 - 44.

[22] Mencik, J.M., Ichchou, M.N.. Multi-mode propagation and diffusion in structures through finite elements. Eur J Mech A/Solids 2005;24(5):877-898.

[23] Souf, M.B., Bareille, O., Ichchou, M., Troclet, B., Haddar, M.. Variability of coupling loss factors through a wave finite element technique. Journal of Sound and Vibration 2013;332(9):2179 - 2190.

[24] Droz, C., Bareille, O., Lainé, J.P., Ichchou, M.N.. Wave-based shm of sandwich structures using cross-sectional waves. Structural Control and Health Monitoring 2018;25(2):e2085.

[25] Zia, Y.B., Khan, A.A., Naushad Alam, M.. Comparison of various higher order shear deformation theories for static and modal analysis of composite beam. In: IOP Conf. Ser. Mater. Sci. Eng.; vol. 377. 2018,

[26] Mencik, J.M.. New advances in the forced response computation of periodic structures using the wave finite element (WFE) method. Comput Mech 2014;54(3):789-801.

[27] Zhong, W.X., Williams, F.W.. On the direct solution of wave propagation for repetitive structures. J Sound Vib 1995;181(3):485-501.

[28] Fan, Y., Collet, M., Ichchou, M., Li, L., Bareille, O., Dimitrijevic, Z.. Energy flow prediction in built-up structures through a hybrid finite element/wave and finite element approach. Mech Syst Signal Process 2016;66-67:137-158.

[29] Mead, D.J.. The forced vibration of one-dimensional multi-coupled periodic structures: An application to finite element analysis. J Sound Vib 2009;319(1-2):282-304.

[30] Droz, C., Bareille, O., Lainé, J.P., Ichchou, M.N.. Diffusion-based design of locally resonant sub-systems using a reduced wave finite element framework. Proceedings of ISMA 2018-International Conference on Noise and Vibration Engineering and USD 2018-International Conference on Uncertainty in Structural Dynamics 2018;:3071-3083.

[31] De Melo Filho, N., Van Belle, L., Claeys, C., Deckers, E., Desmet, W.. On the use of the dynamic mass of metamaterials to calculate the transmission loss based on the acoustic mass law. 13th Int Conf Theor Comput Acoust ICTCA 2017 2017;2017-July.

[32] Li, X.Y., Zhao, X., Li, Y.H.. Green's functions of the forced vibration of Timoshenko beams with damping effect. J Sound Vib 2014;333(6):1781-1795. 